\title{
Experimental Characterization of Wheel-Rail Contact Patch Evolution
}

\section{B. Marshall}

R. Lewis
R. S. Dwyer-Joyce
Department of Mechanical Engineering,
University of Sheffield,
Mappin Street,
S1 JJD, UK
U. Olofsson
S. Björklund
Department of Machine Design,
KTH,
SE 100 44 Stockholm,
Sweden

The contact area and pressure distribution in a wheel/rail contact is essential information required in any fatigue or wear calculations to determine design life, re-grinding, and maintenance schedules. As wheel or rail wear or surface damage takes place the contact patch size and shape will change. This leads to a redistribution of the contact stresses. The aim of this work was to use ultrasound to nondestructively quantify the stress distribution in new, worn, and damaged wheel-rail contacts. The response of a wheel/rail interface to an ultrasonic wave can be modeled as a spring. If the contact pressure is high the interface is very stiff, with few air gaps, and allows the transmission of an ultrasonic sound wave. If the pressure is low, interfacial stiffness is lower and almost all the ultrasound is reflected. A quasistatic spring model was used to determine maps of contact stiffness from wheel/rail ultrasonic reflection data. Pressure was then determined using a parallel calibration experiment. Three different contacts were investigated; those resulting from unused, worn, and sand damaged wheel and rail specimens. Measured contact pressure distributions are compared to those determined using elastic analytical and numerical elastic-plastic solutions. Unused as-machined contact surfaces had similar contact areas to predicted elastic Hertzian solutions. However, within the contact patch, the numerical models better reproduced the stress distribution, as they incorporated real surface roughness effects. The worn surfaces were smoother and more conformal, resulting in a larger contact patch and lower contact stress. Sand damaged surfaces were extremely rough and resulted in highly fragmented contact regions and high local contact stress. [DOI: 10.1115/1.2197523]

Keywords: wheel/rail contact, ultrasound, contact pressure, real area of contact, ultrasonic reflection

\section{Introduction}

Knowledge of contact pressures in the wheel/rail interface is essential information required in both fatigue and wear calculations for wheels and rails. In turn this information is needed when determining design life, re-grinding, and maintenance schedules. Newly machined wheel and rail surfaces are rough. As wear takes place the surfaces become much smoother. However, the position of the contact patch varies with respect to both the wheel and the rail. This means that a long wavelength surface form is also created on both the wheel and rail surfaces. Both the short wavelength surface smoothing and the long wavelength surface roughening will change the size and shape of the area of contact. This in turn will change the contact stress history experienced by both the wheel and rail. Sand is commonly applied to the rail to enhance traction in certain regions of the network. Sand particles become entrained into the wheel rail contact, fracture, and the fragments indent the surfaces. Whilst sand does enhance the traction it does so by increasing the deformation component of friction. The sand particles plough the rail or wheel surface [1] and result in a very rough surface. The damaged surfaces, when they again come into contact, will result in a fragmented area of contact and high local contact stress.

There is currently no definitive experimental technique for determining the size and stress distribution within the aforementioned contact. Currently, emphasis is placed on analytical and computer based numerical models of the problem (see, for ex-

${ }^{1}$ Corresponding author

Contributed by the Tribology Division of ASME for publication in the JouRnAL OF TRIBOLOGY. Manuscript received April 7, 2005; final manuscript received March 21, 2006. Review conducted by Kwangjin Lee. ample, Refs. [2,3]) and the industry relies heavily on such predictions. For smooth surfaces in elastic contact the models work well. But when roughness effects are incorporated the results tend to depend on sampling interval and in addition plasticity effects must be included. Clearly an experimental based method is required not only to explore the contact patch evolution, but also to interpret and validate the numerical models used. This study aims to quantify the size and distribution of the stress in the wheel/rail contact by means of an ultrasonic reflection based method. The approach is then applied to new, worn, and damaged wheel rail contact pairs.

A method has been established by which contact pressures can be determined noninvasively in real engineering components [4]. A similar technique has been applied successfully to measure a single contact between a wheel and rail specimen [5]. In this work, the method is used to study higher loadings, multiple contacts, and the evolution of the contact patch with wear and surface damage. Experimental results are compared to a numerical model, which uses a boundary element method, along with measured wheel and rail surfaces as data inputs.

\section{Ultrasound and Rough Surface Contacts}

When an ultrasonic wave is incident at an incomplete interface between two surfaces, partial reflection occurs (Fig. 1(a)). The sound wave is transmitted at the asperity contacts and reflected from the air gaps. The reflection coefficient, $R$, is defined as the proportion of the incident signal amplitude reflected from the interface.

When the ultrasonic wavelength is long compared to the magnitude of the air gaps, the interface as a whole behaves like a reflector (i.e., individual air gaps do not scatter the sound wave). 


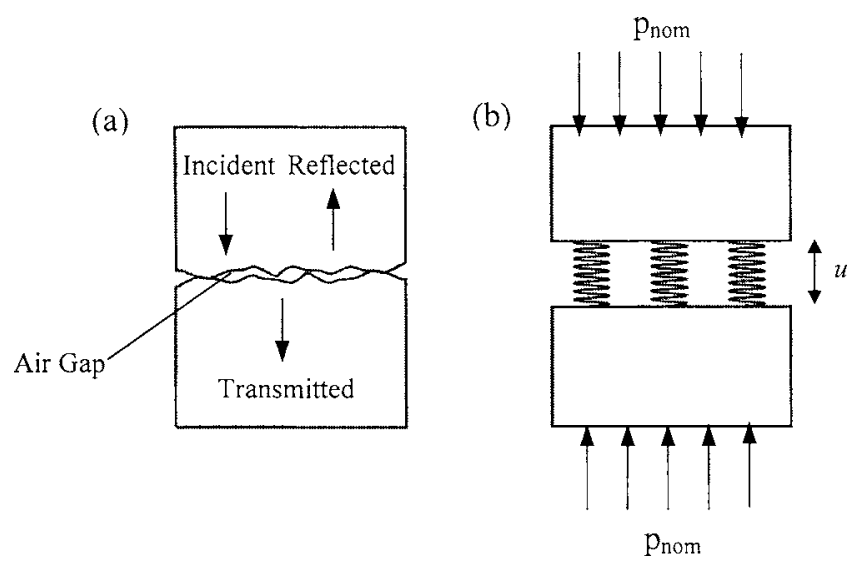

Fig. 1 Schematic diagram of (a) partial reflection of ultrasound at a rough surface contact; and $(b)$ the "spring model" used as a method to determine ultrasonic response of the interface

This case has been investigated and it was found that reflection is governed by the spring-like behavior of the interface [6,7]. For two similar materials in contact there exists a simple relationship between the reflection coefficient and the interfacial stiffness, $K$ :

$$
|R|=\frac{1}{\sqrt{1+(2 K / \omega z)^{2}}}
$$

where $\omega$ is the angular frequency $(=2 \pi f)$ of the ultrasonic wave, and $z$ is the acoustic impedance of the materials in contact (the product of wave speed and density).

The applicability of the spring model to ultrasonic reflection data from a series of rough surface contacts has been assessed [8]. It was demonstrated that the model may be applied to ultrasonic reflection data up to frequencies of about $50 \mathrm{MHz}$, and that it can also be used to determine information about the nature of the contact at the interface.

The stiffness $K$ of a dry interface (expressed per unit area) is defined as the change in nominal contact pressure, $p_{\text {nom }}$, required to cause unit approach of the mean lines of the surfaces. Thus,

$$
K=-\frac{d p_{\text {nom }}}{d u}
$$

where $u$ is the separation of the mean lines of roughness of the two surfaces. Figure $1(b)$ shows a real engineering surface represented using the spring model.

For a given pair of contacting surfaces the interface stiffness depends on the load applied, and hence the contact pressure between them. When the load at the interface is increased the surfaces are pressed closer together with more asperities contacting, thus the stiffness rises. The stiffness in this way is nonlinear and may vary from zero when the surfaces are just touching, to infinity when the surfaces are completely conformal. When the loading occurs the asperity contacts will plastically deform. However, the amplitude and frequency of the ultrasonic wave are such that the deformation is entirely elastic. This means that whilst the shape of the contact interface is determined by plastic flow process, the stiffness obtained ultrasonically (using Eqs. (1) and (2) will be an elastic stiffness.

Maps of the stiffness of an interface can give useful qualitative information about the contact. However contact pressure is more useful. Unfortunately, the stiffness of a rough surface interface depends on the number, size, and distribution of the asperity contacts within it [6]. A contact consisting of several distributed micro-contact regions will be stiffer than one where the individual regions are more closely packed. Therefore, there is no unique relation between stiffness and contact pressure. However, a cali-

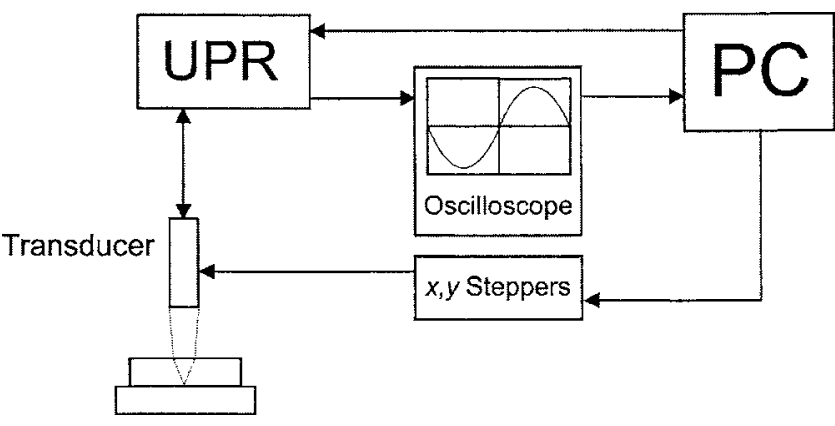

Fig. 2 Schematic of the ultrasonic pulsing/receiving apparatus and scanning system

bration experiment can be performed to find the relation between the two variables for a given rough surface pair [4]. Further, it has been found that for low loads contact pressure and interfacial stiffness were linearly proportional $[9,10]$. This also provides an independent means to obtain contact pressure from stiffness measurements.

\section{Experimental Details}

Ultrasonic Scanning Apparatus. The test equipment consists of a transducer, an oscilloscope, an ultrasonic pulser-receiver (UPR), and a desktop PC. A schematic of the equipment is shown in Fig. 2, with a sample interface included under inspection. The PC emits a control signal to the UPR, which in turn triggers the transducer. The active part of an ultrasonic transducer is a piezoelectric element, which emits an ultrasonic signal in response to an electrical excitation. It is by exciting this crystal that the UPR triggers the transducer. A concave lens is bonded to the piezoelectric element to focus the emitted wave. An ultrasonic pulse contains multiple sound frequencies, with the one containing the maximum energy termed the center frequency of the probe. The contact is investigated by measuring the reflected signals from it, and the same transducer that emitted the signal receives these.

The transducer is positioned in a water bath above the specimen so as to focus the ultrasound onto the interface. Focusing occurs in both the water and the upper component of the specimen. The water also acts as a couplant between the probe and the test specimen, and is required since the high frequency sound wave is scattered by air particles. Both the emitted and reflected signals are displayed on the oscilloscope, from which they can be downloaded to the PC for data processing.

In order to investigate complete contacts, the transducer was mounted on a table automated for $x, y$ scanning. Stepper motors linked to the PC controlled the position of the transducer. In this way, ultrasonic readings were recorded at discrete points over a prescribed area. The vertical position of the transducer was set manually with a screw micrometer.

Transducers and Focusing. In this work a $10 \mathrm{MHz}$ spherical focusing transducer was used to investigate the wheel/rail contact. It had a center frequency of $8.8 \mathrm{MHz}$, and it was at this frequency that the maximum energy content of the signal occurred. The bandwidth of the transducer can also be defined. This is the range of frequencies over which the energy content is $50 \%$ or more of that at the center frequency. It thus has an upper and lower value, and these were 6 and $11.5 \mathrm{MHz}$, respectively, for the $10 \mathrm{MHz}$ transducer. The bandwidth gives the range of frequencies over which information about the contact can be detected. It should be noted that the $50 \%$ drop in signal strength corresponds to a drop of $6 \mathrm{~dB}$.

The ultrasonic signal is focused onto the interface by refraction. At the front of the transducer is a concave lens, which provides the initial convergence of the sound wave. The ultrasound then converges further as it passes through the water couplant before 


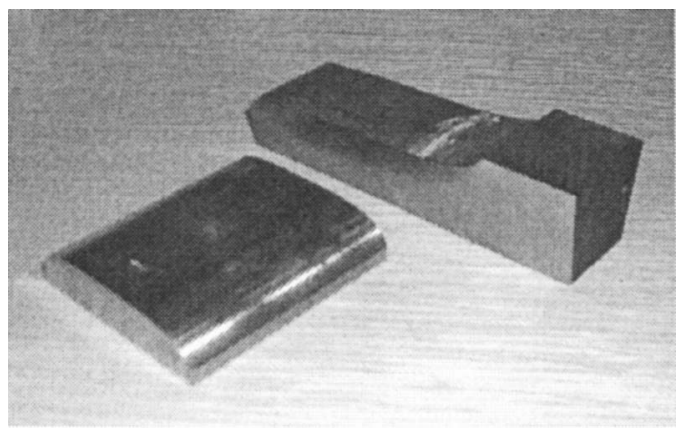

Fig. 3 Photograph of contact specimens cut from a worn wheel and rail

refracting once more at the upper boundary of the test specimen. It then focuses down to a spot in the component under inspection. The refraction at a boundary is defined as

$$
\frac{\sin \theta_{i}}{c_{1}}=\frac{\sin \theta_{r}}{c_{2}}
$$

where $\theta_{i}$ and $\theta_{r}$ denote the angles of incidence and refraction of the sound wave at the boundary, and $c_{1}$ and $c_{2}$ the speed of sound in the material before and after the interface, respectively. Using Eq. (3) the refraction and subsequent change in focusing angle of the ultrasound can be determined as it passes from the water couplant into the specimen. The length of the water bath required to focus the ultrasound onto the interface can then be calculated, and the transducer position adjusted accordingly.

The ultrasound focuses down to a finite spot. This spot diameter, $d$, is the resolution of the ultrasonic technique, and is expressed as follows [11]:

$$
d(-6 \mathrm{~dB})=\frac{1.028 l_{w} c_{w}}{1000 f D}
$$

where $l_{w}$ is the focal length in water, $c_{w}$ is the speed of sound in water, $f$ the ultrasonic frequency, and $D$ is the transducer element diameter.

It is essential when choosing a transducer for a given application that the spot diameter is minimized, as this maximizes the resolution of the ultrasonic technique. In this work the focused spot size of the transducer used was approximately $1 \mathrm{~mm}$. Contact pressure readings determined by this ultrasonic approach are
Table 1 Wheel/rail specimen surface roughness values

\begin{tabular}{lcc}
\hline \hline Wheel/rail contact & $\begin{array}{c}\text { Specimen } \\
\text { component }\end{array}$ & $\begin{array}{c}\text { Mean surface roughness } \\
(\mu \mathrm{m})\end{array}$ \\
\hline Unused & Wheel & 4.11 \\
& Rail & 2.65 \\
Sand damaged & Wheel & 12.45 \\
& Rail & 20.38 \\
Worn-tread & Wheel & 4.57 \\
& Rail & 0.69 \\
Worn-flange fillet & Wheel & 5.51 \\
& Rail & 0.86 \\
\hline
\end{tabular}

therefore averages over this region (in fact the transducer response function is a $\sin x / x$ shape, so there is a center weighting to the signal, and the spot size represents a conservative estimate of the resolution)

Test Samples and Loading. Sample wheel and rail specimens were cut from actual wheel and rail sections (as shown in Fig. 3). The wheel sections were R8T and the rail sections UIC60 900A. Three sets of specimens were used; unused factory machined, worn, and sand damaged wheel and rail sections. The worn wheel specimen was from an X10 trailing wagon used in Stockholm local traffic, and was removed after a traveled distance of approximately $150,000 \mathrm{~km}$ and the worn rail section was from a $346 \mathrm{~m}$ radius curve also from the Stockholm line (more details on the amount of traffic passing over the rail as well as hardness profiles can be found in Refs. $[12,13])$. The sand damaged specimens were from wheel and rail section that had sustained severe surface damage during tests to ascertain the effect on wear of adhesion sanding [1].

Table 1 shows the mean surface roughness values for the different wheel/rail specimens investigated. The roughness measurements (center line average, $R_{a}$ ) are shown at two points for each worn specimen, this is because a twin contact was observed that is detailed subsequently in the Results section. Roughness profiles for the unused and sand damaged wheel and rail specimens are also shown in Fig. 4. These clearly illustrate the different surface characteristics. The rail specimens exhibited similar relative levels of roughness. Vickers hardness measurements were also recorded for each of the specimens. As the spread of the hardness results was minimal, average values for the two materials are used; these are 378 and $242 \mathrm{Hv}$ for the wheel and rail, respectively.

In this series of experiments the contact was normally loaded in (a)

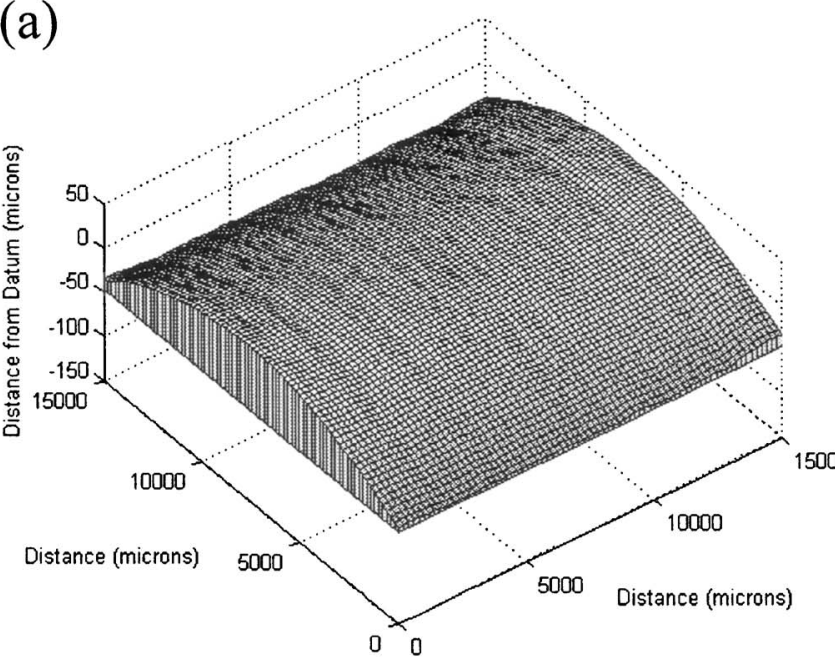

(b)

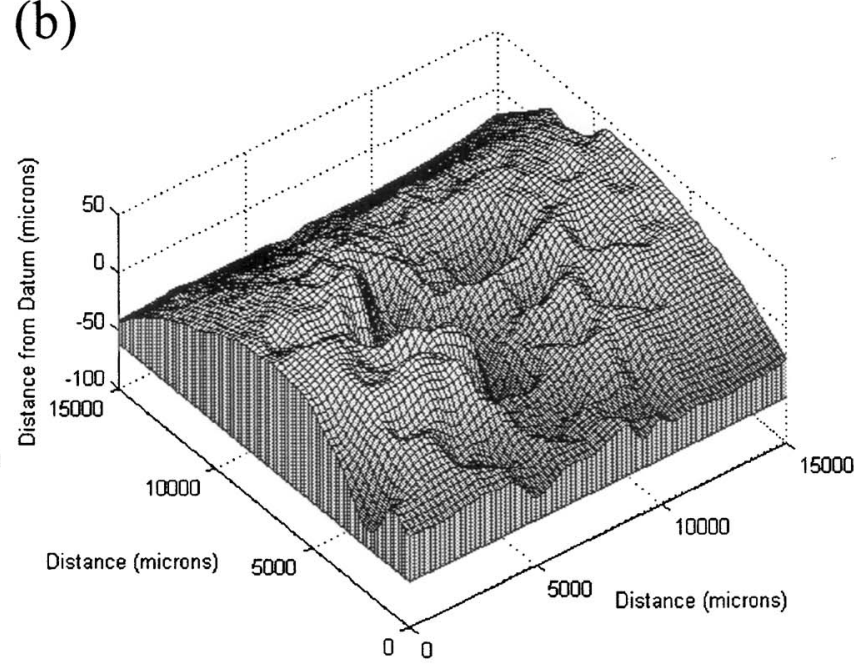

Fig. 4 Wheel surface profiles for (a) unused specimen and (b) sand damaged specimen. Displayed on the same horizontal and vertical scales. 


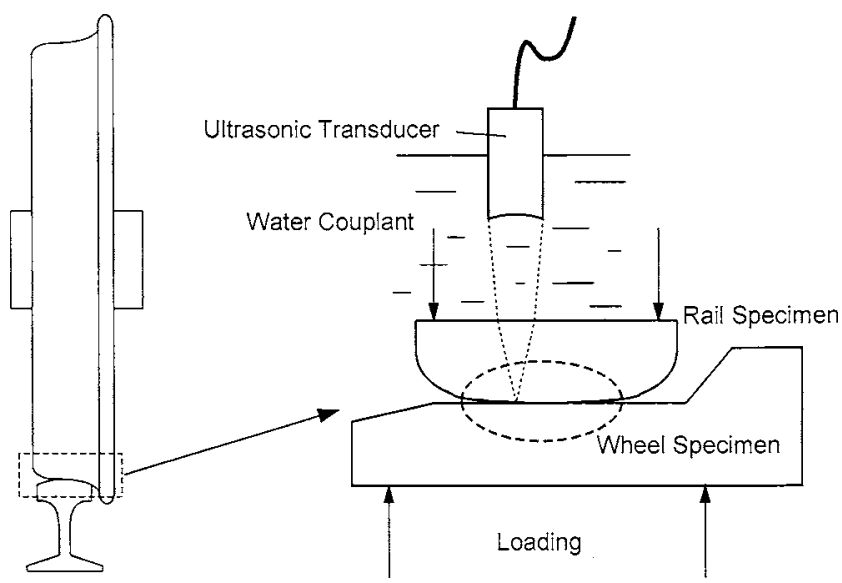

Fig. 5 Schematic diagram of the scanning of a wheel/rail contact

the range $20-80 \mathrm{kN}$, this is typical of a normal loading these specimens would expect in service [14]. The specimens were loaded together in a purpose built frame, and the loading applied using an Enerpac hydraulic loading jack.

Scanning Procedure and Signal Processing. Figure 5 shows the loaded interface between the specimens being scanned. The transducer was scanned over the contact area, with the amplitude of the reflected ultrasonic pulse recorded at $0.25 \mathrm{~mm}$ intervals. The reflected signal from the interface has a lower value than the emitted pulse for the two following reasons; partial transmission of the signal takes place at the interface, and attenuation occurs in the material bulk. A reference trace was also taken from a point at the side of the interface where the wheel and rail were not in contact. The reference signal is only diminished by attenuation, as all the ultrasound is reflected from the steel-air interface at this point. If the amplitude of the reflected signal from the contact is divided by the amplitude of the reference trace then the attenuation is canceled out. This leaves the fraction of ultrasound incident at the interface that is reflected from it, in other words the reflection coefficient, $R$. In this way, reflection coefficient scans of the interface at each loading were constructed using the reflected voltage data. Applying Eq. (1) to the reflection coefficient data produced interfacial stiffness maps of the contact.

The technique presented here is a time domain method. In using it, an assumption is made that the peak amplitude occurs at the center frequency for both the incident and reflected pulses. Although more time consuming, it is possible to analyze the reflection data in the frequency domain [8]. The reflected and reference pulses are downloaded and converted to the frequency domain using a fast Fourier transform (FFT). The reflection coefficient is then obtained as a function of ultrasonic frequency by dividing the reflected FFT by the reference one. The spring model can then be applied to the reflection data to find the interfacial stiffness at a range of frequencies. Interfacial stiffness is independent of ultrasonic frequency, and can then be determined from the calculated data. The time domain method has been used in this study as it represents a large time saving when repeatedly scanning large contact patches.

Calibration Experiment. A calibration experiment was performed to find the relationship between contact pressure and interfacial stiffness for each of the different wheel/rail systems. Figure 6 shows the calibration specimens and the experimental setup.

The calibration specimens were machined from the same material and with the same surface finishing process as the wheel/rail components (for both the unused and damaged specimens). Recreating the same surface finish is critical as the amount of ultrasound reflected from a contact is clearly affected by the surface

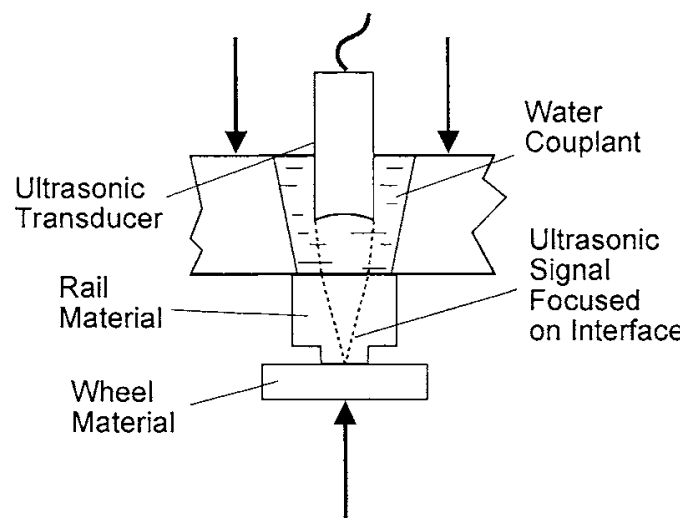

Fig. 6 Schematic diagram of the calibration specimens and experimental setup

roughness of the contacting specimens $[15,16]$. In practice this proved difficult, the calibration specimens were repeatedly ground and the surface profiles measured after each process to achieve as close as possible a match. Whilst this method was at best approximate, it appeared to give good results when the calibration was independently checked (described later and demonstrated in Fig. $15)$.

The specimens were hydraulically loaded together, and a series of single point ultrasonic readings taken for known loads. A reference signal was also recorded, this time with the lower disc absent. Reflection coefficient and interfacial stiffness (using Eq. (1)) were then calculated at each loading. As the interface is flat and of known geometry, contact pressures were determined by dividing load by nominal area of contact. Figure 7 shows the resulting relationship between the contact pressure and interface stiffness. For a contact pair of high surface roughness, a greater pressure is required to achieve a certain surface conformity and hence stiffness. The rougher contact pairs result in a shallower slope on the stiffness versus pressure plot. The data of Fig. 7 is largely consistent with the roughness data of Table 1 . However, the effect of sand damage to the interface stiffness is less severe then the unused specimens. This is possible because the roughness of the sand damaged surfaces is of relatively long wavelength and therefore the surfaces can conform to some degree.

When the worn specimens were loaded together a twin contact was observed, with the patch consisting of both tread and flange fillet contact zones. Although the double contact is not discussed here it should be noted that separate calibration experiments were performed for the two contact zones, due to their differing surface roughness.

Over the pressure range shown in Fig. 7 the stiffness-pressure relationships were approximated as linear. At very high contact pressures the stiffness will tend to infinity as the surfaces approach complete conformity. But over the range tested here the data appear to be close to linear. At the higher pressures the error is in the region of $10-15 \%$. This will clearly cause the same error in the predicted pressure measurement. Whilst most of the data gathered from the wheel rail contacts are at low pressure, the higher-pressure regions are subject to this magnitude of error.

The relationship $p=c K$ was fitted to the data, where $p$ is the contact pressure, $K$ the interfacial stiffness, and $c$ the reciprocal of the gradient of the line. The values of $c$ were 291, 263, 123, and 198 for the unused, sand damaged, worn tread, and worn flange fillet contacts, respectively. The curves of Fig. 7 can then be used as calibration curves to determine the local contact pressure from a stiffness map recorded for a wheel-rail contact.

This calibration technique is an experimental method only. Therefore, the calibration specimens must be subjected to the same load conditions as the wheel/rail components the calibration is required for. The wheel/rail specimens have experienced many 


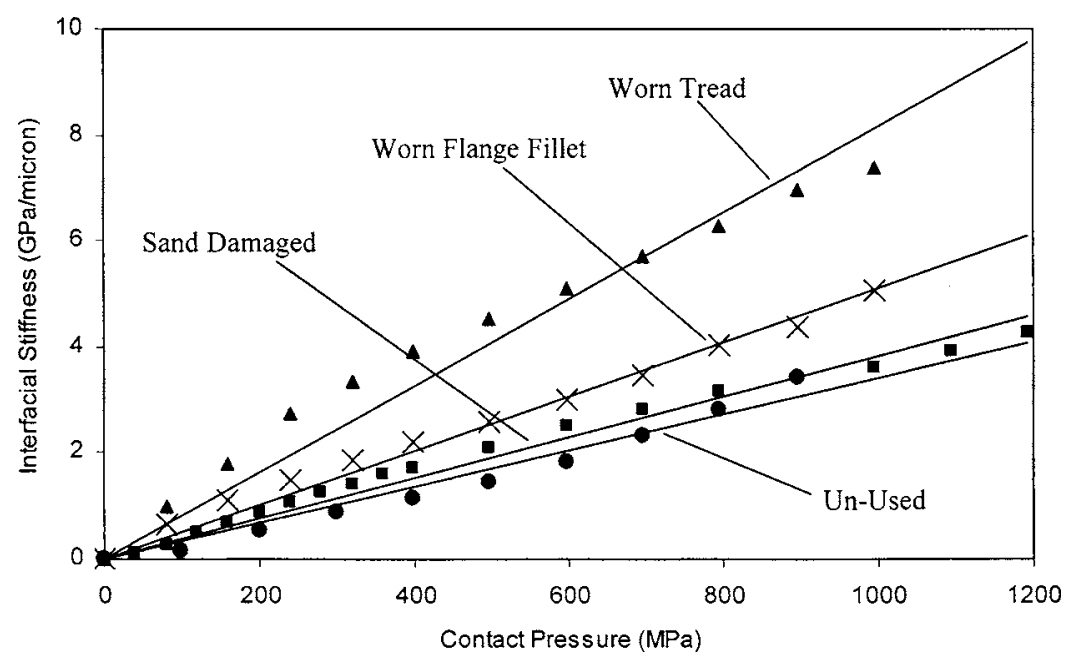

Fig. 7 Relationship between interfacial stiffness and contact pressure for various wheel-rail specimen pairs. These curves are used to calibrate stiffness maps to produce pressure distributions.

previous loadings. When two surfaces are loaded together during the first load cycle, behavior is elastic, and then plastic in subsequent cycles. In this case we have a first loading of a surface that has experienced many previous loads. Thus, a similar load regime was employed on the calibration specimens to accurately model this situation.

\section{Ultrasonic Results}

Unused Wheel/Rail Specimens. Figure 8 shows the contact pressure maps for the unused wheel/rail contact at loadings between 40 and $80 \mathrm{kN}$. Also marked in Fig. 8 are the predicted Hertzian elastic contact patches for the two bodies (see Ref. [17] for calculation).

As shown, there is good geometric agreement between the analytically predicted and measured contact patches for the unused specimens. However, when comparing to Hertz, the measured contacts are to a small degree fragmented. This is due to an initial surface roughness present on the specimens. This produces a less conformal contact, leading to a larger nominal contact area and higher measured maximum contact pressures than those predicted by theory (see Table 2). The measured peak pressures are above the maximum calibrated value $(1200 \mathrm{MPa})$. Thus, the calibration has been extrapolated to include these higher peak values. However, the percentage of the contact over this threshold is small, and the relation between stiffness and pressure is tentatively assumed linear at this point.

From the hardness measurements of the specimens the yield stress of the contact patch was estimated [18]. The rail material was found to yield first, giving an onset of plastic behavior at a contact stress of $850 \mathrm{MPa}$ in the patch. Given this information, the percentage of the total area of the contact in excess of the yield stress at each loading was determined. Figure 9 shows the elastic and plastic analysis of the contact patch at a series of loads; also included is the Hertzian contact area, expressed as a percentage of the measured total area of contact.

As shown by Fig. 9 the fraction of plasticity in the contact grows moderately with increasing load. Also, the Hertzian predicted contact geometry remains between 60 and $70 \%$ of the mea-
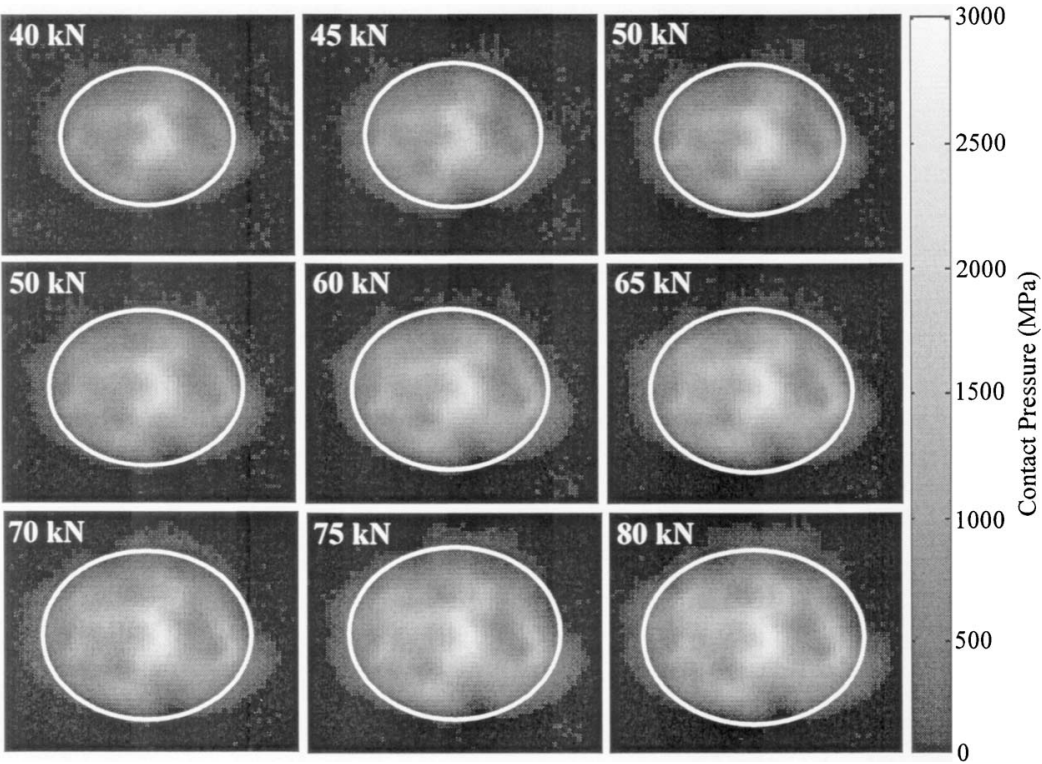

Fig. 8 Ultrasonic contact pressure maps for the unused wheel/rail contact 
Table 2 Measured and predicted maximum pressures for the unused wheel and rail specimens

\begin{tabular}{ccccc}
\hline \hline & $\begin{array}{c}\text { Predicted elastic } \\
\text { contact area } \\
\left(\mathrm{mm}^{2}\right)\end{array}$ & $\begin{array}{c}\text { Measured } \\
\text { contact area } \\
\left(\mathrm{mm}^{2}\right)\end{array}$ & $\begin{array}{c}\text { Predicted } p_{\max } \\
(\mathrm{MPa})\end{array}$ & $\begin{array}{c}\text { Measured } p_{\max } \\
(\mathrm{MPa})\end{array}$ \\
\hline 40 & 52.1 & 86.6 & 787 & 1991 \\
45 & 56.5 & 86.2 & 819 & 2050 \\
50 & 60.6 & 89.0 & 848 & 2130 \\
55 & 64.6 & 92.6 & 875 & 2224 \\
60 & 68.5 & 93.6 & 901 & 2421 \\
65 & 72.2 & 96.0 & 925 & 2418 \\
70 & 75.8 & 101.9 & 948 & 2538 \\
75 & 79.4 & 107.1 & 970 & 2585 \\
80 & 82.4 & 111.9 & 992 & 2628 \\
\hline \hline
\end{tabular}

sured contact area. Thus the rate of growth of the total area of contact is similar to that predicted by Hertz over the range investigated, with the overall size differing due to the roughness effects.

It should be noted that these are predictions of the contact patch on a section of unused rail. In service the rail will be subjected to shakedown caused by repeated rolling and sliding. This will cause the build up of a strain hardened near surface layer. The yield stress would then be higher than that which has been used in this analysis. Also the surfaces would wear to a more conformal shape. Both effects would cause there to be less plastic deformation in a run-in rail. Table 2 and Figs. 8 and 9 essentially show the levels of stress and plasticity that would be observed during the first contact between the wheel and rail.

Worn and Damaged Wheel/Rail Specimens. Figure 10 shows the contact patch from the unused, sand damaged, and worn wheel/rail interfaces at a load of $65 \mathrm{kN}$. A Hertzian contact analysis has not been performed for the sand damaged and worn cases due to the nonconstant radii of curvature of the specimens. When comparing the contact patches from the unused specimens to those from the sand damaged interface there are marked differences. Although the load supported is similar, the contact patch is extremely fragmented. The sand damage leads to an extremely rough surface and a generally patchy contact. There are now large areas within the contact patch where the wheel and rail are no longer touching, leading to a spread in the contact. The peak pressure within the contact patch is also reduced. This is because the pressures ultrasonically recorded are mean values measured over the ultrasonic spot, and are reduced by the increased spread of the contact. They are not those on an asperity scale, which may be higher for a sand damaged surface.

The worn wheel/rail specimens also give a different result when compared to the unused case. Both the wheel and rail become worn in use, leaving a smooth polished surface on both specimens. However, because of the many different relative positions between the wheel and rail in a train's operation, the wear occurs over a large area of the surface. Waviness is introduced on the wheel surface, and its effect is evident in the worn contact patch shown in Fig. 10(c). On a global scale the wear causes the relative radii of the two surfaces to change. Figure 11 shows the measured

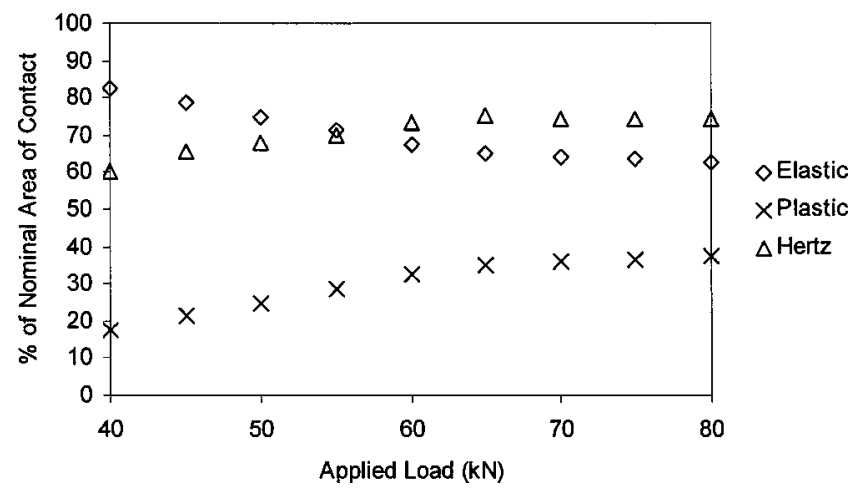

Fig. 9 The proportion of elastic and plastic contact within the nominal region of wheel-rail contact

surface roughness profiles (recorded on a profilometer with no post-filtering of the data) for the worn and unused wheel surfaces. The wheel hollows and the contact radius changes significantly. This results in a larger radius in the transverse direction, and hence results in a more circular contact patch (as shown schematically in Fig. 11).

In addition to the long wavelength roughness change, the worn surfaces change on a short wavelength. The worn surfaces are smoother (i.e., the turning and grinding marks are worn away leaving a polished surface). This leads to a more conformal contact on an asperity scale. Both these effects cause the peak pressure to be reduced when compared to the unused specimens.

Table 3 shows the analysis of the three different contact patches. Both the absolute values of elastic and plastic contact areas are given, along with the elastic and plastic areas as a percentage of the total area of contact. The yield stress has been obtained from the hardness of the contact surface determined using a micro-indentor. So this value corresponds to the strain hardened surface layer of run-in rail and is higher than that for unused rail. For this reason, and the fact that the surfaces are smoother and more closely conforming, the proportion of plastic contact is lower.

As shown, the sand damage increases the total area of contact when compared to the unused specimens, causing the overall plastic zone to reduce and make up a lower percentage of the overall contact area. In the case of the worn specimens the total area of contact again increases. When compared to the unused specimens the plastic zone is again reduced making up a lower percentage of the contact.

Figure 12 shows the distribution of the yield zone in the three different wheel/rail contacts. As shown, for the sand damaged case the position of the yield zone is dependent on the local removal of material from the surface. Similarly in the worn case the plastic zone is affected by the nature of the wear. In this case the wear causes an imbalance in the load distribution in the contact, giving the observed off-center plastic zone.

Twin Contacts. If severe wear occurs to a wheel flange in
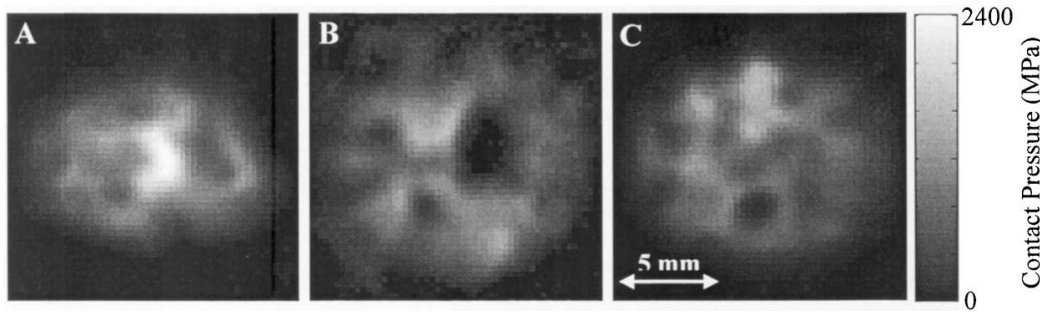

Fig. 10 Wheel rail contact at $65 \mathrm{kN}$ for (a) unused, (b) sand damaged, and (c) worn specimens 
(a)
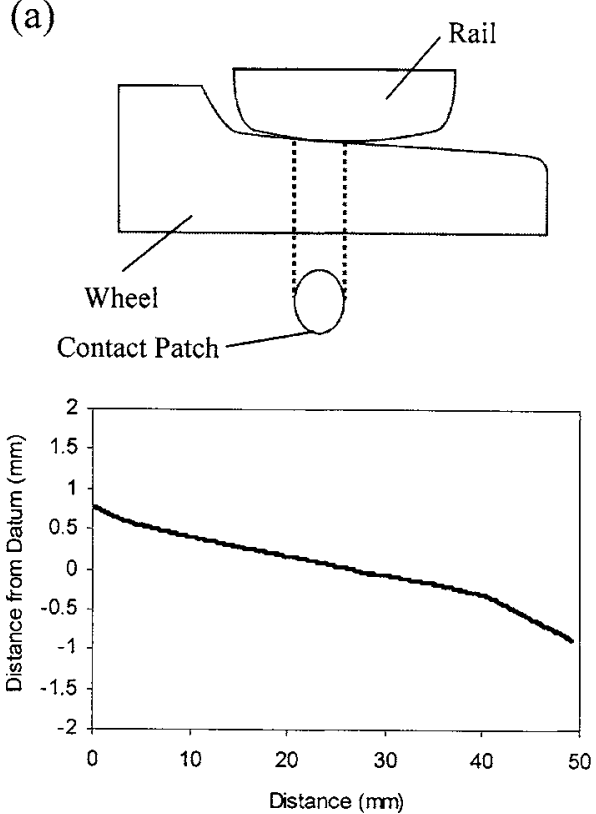

(b)
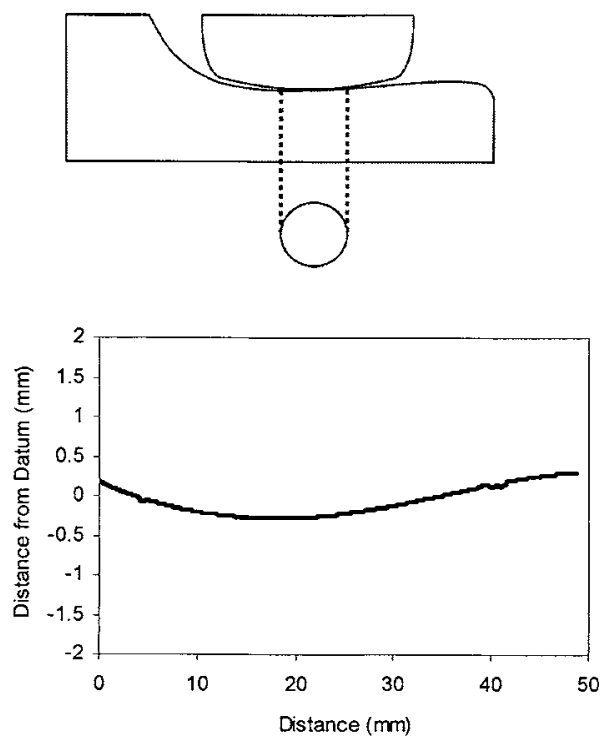

Fig. 11 Schematic diagram showing the contact between (a) worn and (b) unused wheel/rail specimens

curves where large lateral forces occur, its profile conforms to that of the rail. This may result in a two-point contact occurring, with a tread contact and another contact at the flange fillet or flange, as shown in Fig. 13. This phenomenon was investigated using the worn wheel and rail specimens. The specimens were relocated so that the rail was contacting over the flange region of the wheel. The resulting pressure map for a double contact at $65 \mathrm{~N}$ is shown in Fig. 14 and compared with that for a single contact at the same load. Details of the areas and pressures for the worn contacts at $65 \mathrm{kN}$ are shown in Table 4.

The introduction of the second contact causes the load supported at the tread to reduce. Conversely, the total area of contact at the tread increases, however this is not significant as the wheel and rail are in a different relative position. As detailed in Table 4, approximately $30 \%$ of the total load is now supported in the second contact, with a similar fraction of the total area of contact occurring there. This reduces the intensity of the peak pressures in the tread contact. As shown in Fig. 14(b) and Table 4 the peak pressure is similar in each component of the double contact. The overall area of contact is also increased for the twin contact when compared to the single case. Due to the concave radius of curvature of the wheel specimen near the flange, the second contact resembles a line. All this is in good qualitative agreement with numerical simulation results for a two-point contact presented in Ref. [2]. As the load is now supported over a greater area, the level of plasticity in the double contact is reduced. Both the absolute value of plastic area, along with the percentage it makes up of the nominal contact area, are smaller when compared to the

Table 3 Measured wheel/rail contact areas, and proportions of elastic and plastic contact

\begin{tabular}{lcccccc}
\hline \hline $\begin{array}{l}\text { Wheel/rail } \\
\text { contact }\end{array}$ & $\begin{array}{c}A_{\text {total }} \\
\left(\mathrm{mm}^{2}\right)\end{array}$ & $\begin{array}{c}A_{\text {elastic }} \\
\left(\mathrm{mm}^{2}\right)\end{array}$ & $\begin{array}{c}A_{\text {plastic }} \\
\left(\mathrm{mm}^{2}\right)\end{array}$ & $\frac{A_{e}}{A_{\text {total }}}$ & $\begin{array}{c}A_{p} \\
A_{\text {total }}\end{array}$ & $\begin{array}{c}p_{\max } \\
(\mathrm{MPa})\end{array}$ \\
\hline Unused & 96.0 & 62.6 & 33.4 & 65.2 & 34.8 & 1283 \\
Sand damaged & 163.9 & 154.5 & 9.4 & 94.3 & 5.7 & 1228 \\
Worn & 142.4 & 117.9 & 24.4 & 82.9 & 17.1 & 1669 \\
\hline \hline
\end{tabular}
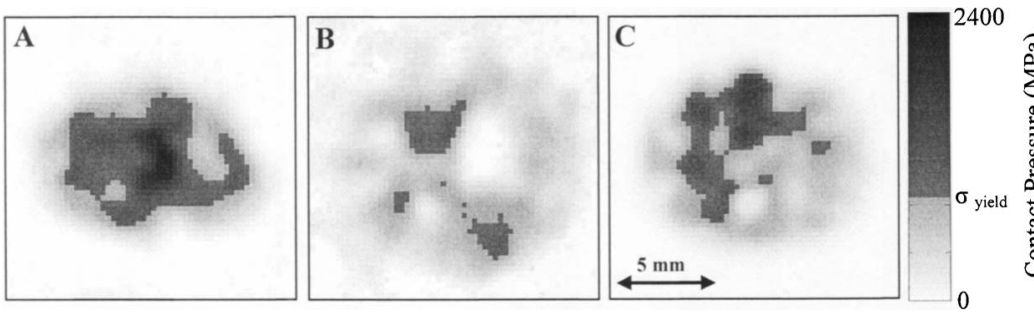

Fig. 12 Wheel/rail contact pressure maps at $65 \mathrm{kN}$ showing the regions of plastic contact for (a) unused, (b) sand damaged, and (c) worn specimens. Two different color maps are used in the scale to distinguish elastic and plastic contact areas. 
(a)

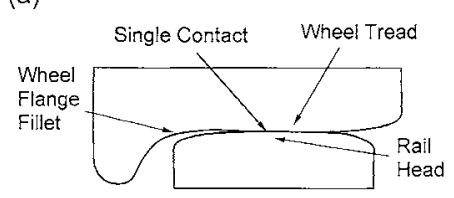

(b)

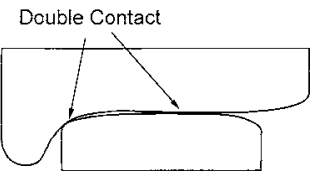

Fig. 13 Single (a) and double (b) wheel/rail contacts

single case. A plastic zone now exists in both the tread and flange components of the double contact, with the larger plastic area occurring in the more heavily loaded tread.

Total Load. A simple check can be made on the validity of the calibration procedure. As the contact pressure distribution has been determined at each loading, it can be summed to find the total load at the interface. The applied hydraulic load can then be compared to the total measured interface load for each scan. As shown in Fig. 15, for the unused and sand damaged wheel/rail contacts the correlation is good between the two methods for determining total load. This indicates that the calibration procedure gives acceptable results.

\section{Numerical Contact Modeling}

The contact between the wheel and rail is compared with a $3 \mathrm{D}$ numerical contact model that uses real digitized surface profiles. Initially a stylus instrument is used to measure the surface topography of an area that is larger than the anticipated contact area of the contacting bodies, and the three-dimensional topography digitized (at a sampling interval of $10 \mu \mathrm{m}$ ).

The digitized topography is then used as an input to a contact computation program [19] that works by replacing the continuous pressure distribution with a discrete set of constant pressure elements. The contact computation applies to normally loaded, frictionless, elastic contacts, modeling the interacting bodies as infinite half-spaces, thus implying that the region of contact is small
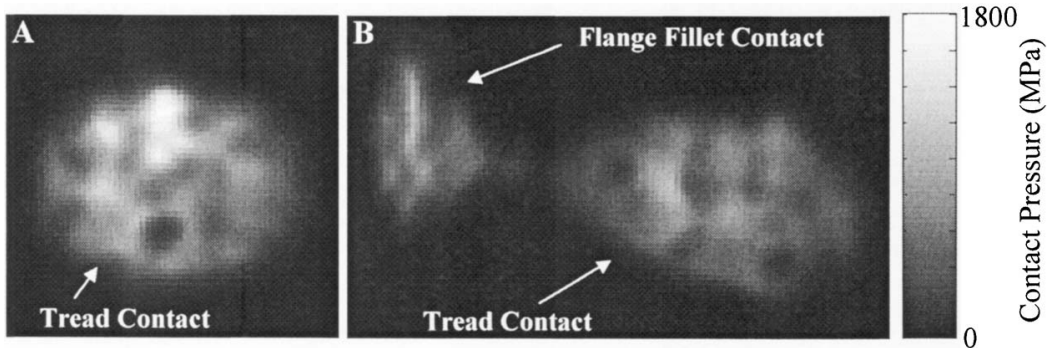

Fig. 14 Worn wheel/rail contact pressure maps at $65 \mathrm{kN}$ for (a) single contact and $(b)$ double contact

Table 4 Wheel/rail contact loading and geometry

\begin{tabular}{lcccccccc}
\hline \hline & Contact & $\begin{array}{c}\text { Measured } \\
\text { load (kN) }\end{array}$ & $\begin{array}{c}A_{\text {total }} \\
\left(\mathrm{mm}^{2}\right)\end{array}$ & $\begin{array}{c}A_{\text {elastic }} \\
\left(\mathrm{mm}^{2}\right)\end{array}$ & $\begin{array}{c}A_{\text {plastic }} \\
\left(\mathrm{mm}^{2}\right)\end{array}$ & $\frac{A_{e}}{A_{\text {total }}}$ & $\frac{A_{p}}{A_{\text {total }}}$ & $\begin{array}{c}p_{\text {max }} \\
(\mathrm{MPa})\end{array}$ \\
\hline Single & Worn & 64 & 142.4 & 117.9 & 24.4 & 82.9 & 17.1 & 1669 \\
Double & $\begin{array}{c}\text { Tread } \\
\text { Flange } \\
\text { fillet } \\
\text { Total }\end{array}$ & 46 & 166.4 & 163.4 & 3.0 & 98.2 & 1.8 & 1144 \\
& 63 & 71.2 & 70.0 & 1.2 & 98.3 & 1.7 & 1114 \\
& 63 & 237.6 & 233.4 & 4.2 & 98.2 & 1.8 & 1144 \\
\hline \hline
\end{tabular}

(a)

(b)
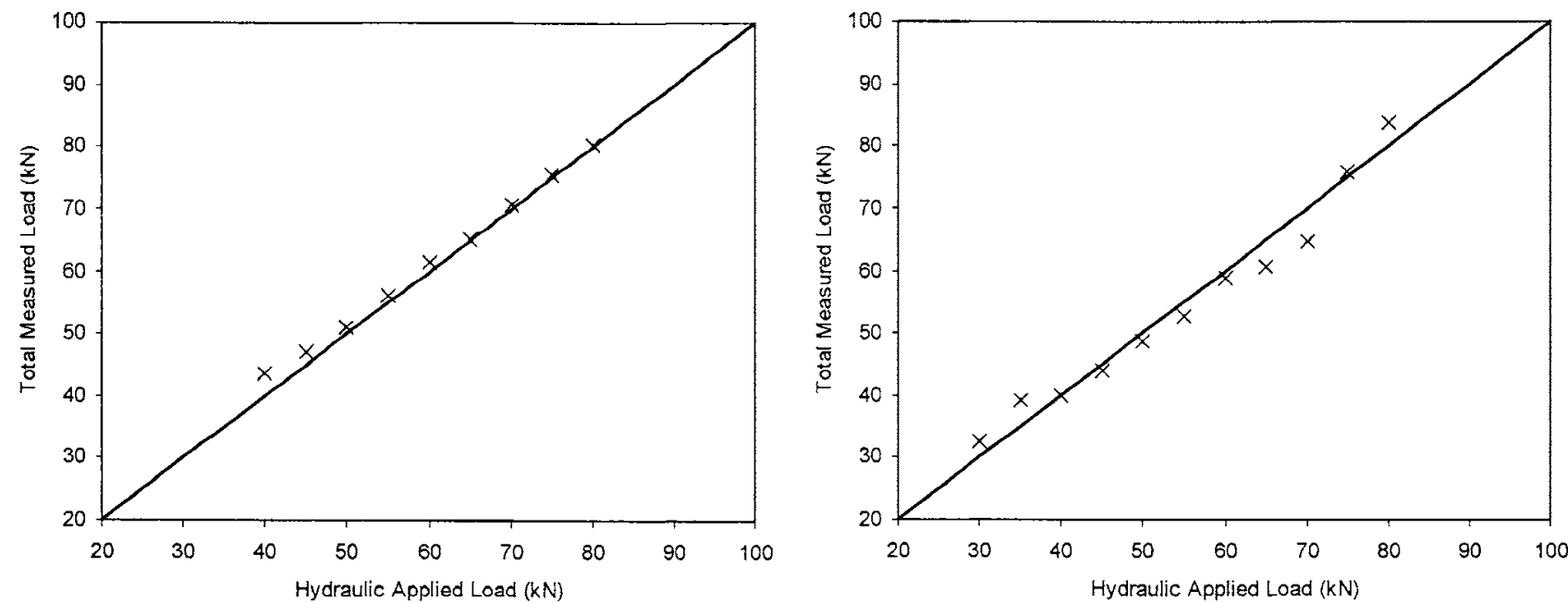

Fig. 15 Applied and measured load comparison; (a) unused specimens and (b) sand damaged specimens 
(a)

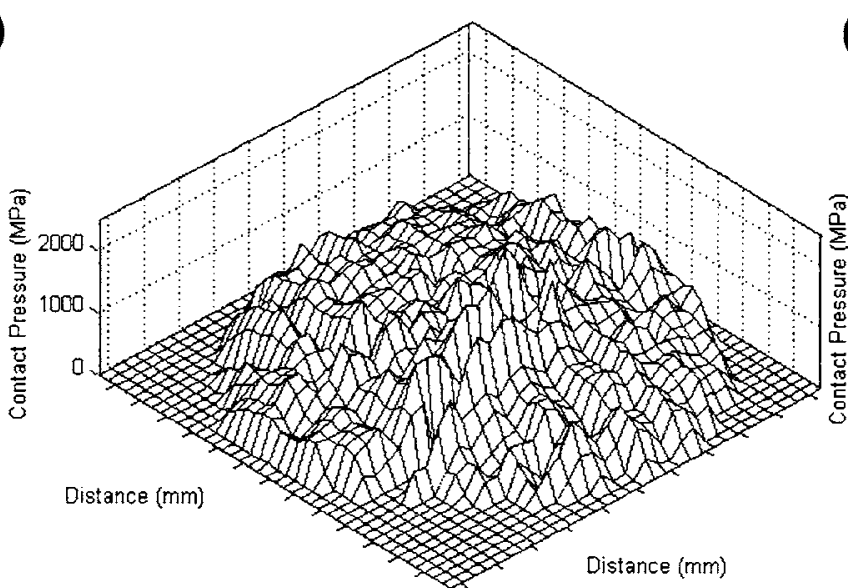

(c)

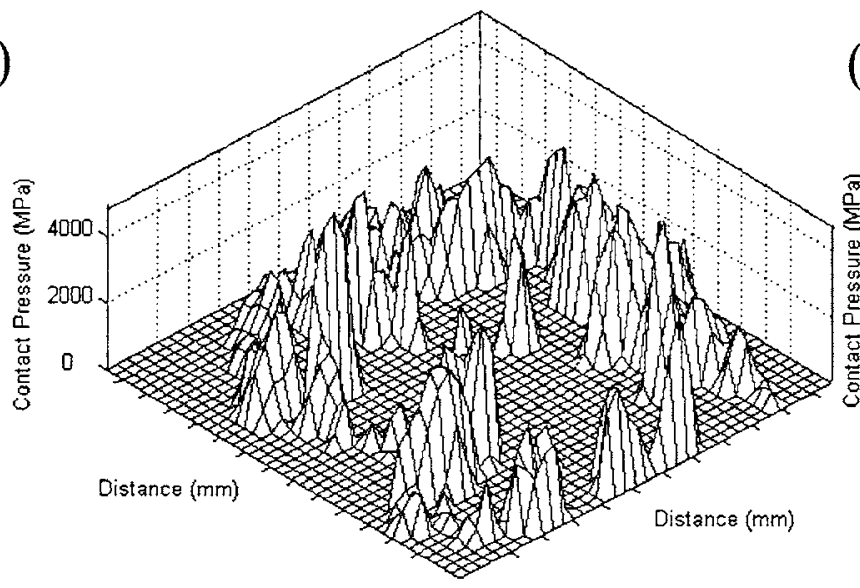

(b)
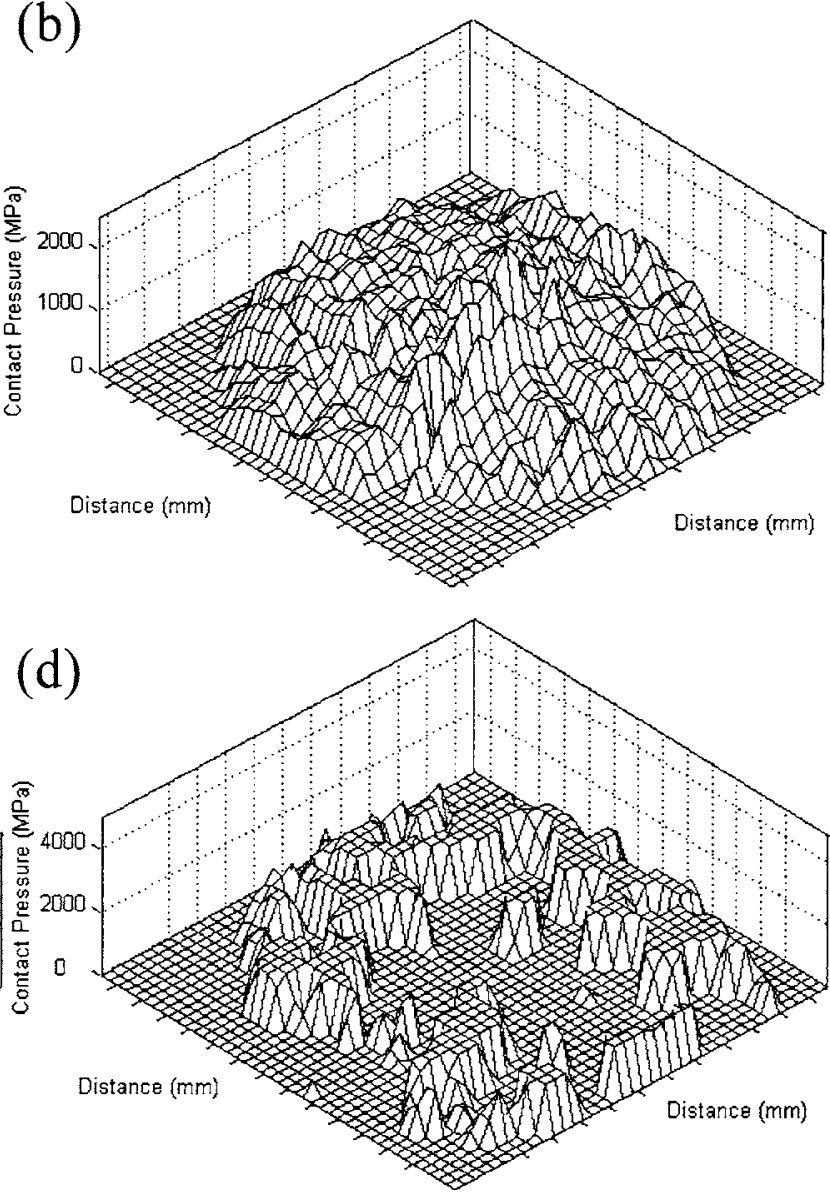

Fig. 16 Numerical contact pressure maps at $80 \mathrm{kN}$ for (a) unused specimens elastic case, (b) unused specimens elastic-plastic case, (c) damaged specimens elastic case, and (d) damaged specimens elastic-plastic case

compared to the size of the bodies. Results were compared for an elastic calculation and also for a modified version of the computation that accounts for some localized plasticity.

Elastic Contact Model. The contact area is divided into $\mathrm{N}$ rectangular cells, each of these being subjected to a uniform pressure. Knowing the gap, $h$, between the cells before deformation and the applied normal displacement $\delta_{z}$, the solution was obtained from an equation system which written in matrix form becomes

$$
[C][p]=\delta_{z}-[h] \equiv[d]
$$

where $C$ is the influence coefficient matrix. Component $C_{i j}$ relates the deformation at cell $i$ due to a unit pressure in cell $j$. The influence coefficients for a uniform pressure on a rectangular cell were found by Love [20]. The sizes and shapes of the real contact areas are not known in advance. An initial estimate, which will contain the true contact region, is the contact area obtained if the bodies are allowed to penetrate each other without any interaction. When solving Eq. (5), the pressures at cells outside the true contact regions become negative. These cells are removed and the equation system is solved iteratively until all pressures are positive. This solution represents the pressures obtained for a purely elastic deformation. The procedure has been carried out for recorded wheel and rail profiles for the unused and sand damaged surfaces. The resulting elastic solutions are shown in Figs. 16(a) and $16(c)$.

Plastic Modification. Many of the cells have predicted pressures well in excess of yield. The model is then modified to account for plasticity at the asperity contact. In contacts between rough surfaces it is often found that the pressure in some cells is excessively large, implying that the deformation is plastic rather than elastic. An approximate method to account for this is to limit the allowable pressure by a yield pressure $P_{y}$. Thus, Eq. (5) is first solved according to the procedure outlined above, removing all cells having negative pressures. The resulting pressures are inspected and all pressures exceeding the yield pressure $P_{y}$ are set to equal the yield pressure and then removed from the subsequent iterations. However, the cells with plastic behavior do still contribute to the deformation at the elastic cells and Eq. (5) thus becomes

$$
\left[C_{e e}\right]\left[p_{e}\right]=\left[d_{e}\right]-\left[C_{e p}\right]\left[p_{p}\right]
$$

where the subscripts $e$ and $p$ contain indices to cells with elastic and plastic behavior respectively. For example, $C_{e p}$ denotes the rows of $C$ that correspond to cells with elastic deformation and the columns that correspond to plastic deformation. Equation (6) is repeatedly solved until all pressures are positive and less or equal to $P_{y}$.

Other researchers [21] have described similar methods to account for plasticity in contact pressure calculations. The method is simple and is only an idealization of the true plastic behavior in rough contacts. It is, however, believed that when the plastic deformations are small and limited to a minor part of the contact area, the approximate method described above is sufficiently accurate for the purposes of this work.

The method depends critically on the selection of a yield pressure. In this study this has been obtained from the surface microhardness and so represents a yield pressure of the material in its existing unused state. Repeated cyclic contacts will induce further deformation and strain hardening. Thus, the model will predict the 

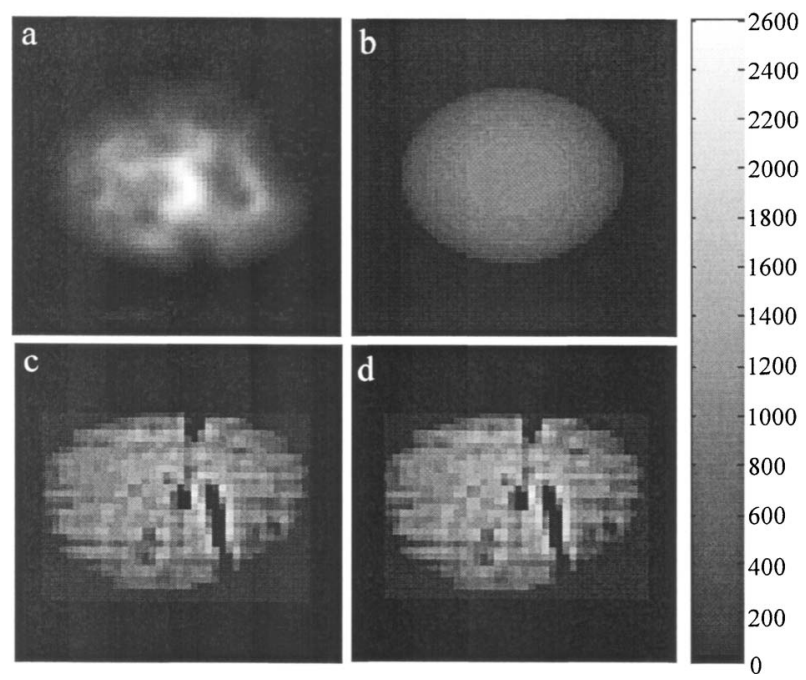

Fig. 17 Unused specimen contact pressure maps for a load of $80 \mathrm{kN}$ for (a) ultrasonic measurement, (b) Hertzian smooth elastic, (c) elastic model, and (d) elastic-plastic model

contact patch stress distribution for a single normal contact, commensurate with the experimental data. Repeated contacts would lead to an elevated yield stress as the material strain hardens and the contact undergoes shakedown.

The model has been rerun with the plasticity analysis for both the unused and damaged wheel and rail specimens. Figures $16(b)$ and $16(d)$ illustrate the results. The peak pressures predicted in the elastic model are greatly reduced when plasticity is incorporated into the model. Fragmentation seen in the ultrasonic measurements is again evident, particularly with the damaged specimens. The technique was not performed for the worn case.

\section{Discussion}

Surface Roughness and Surface Form Evolution. The new unused wheel and rail specimens are relatively rough. The turning and grinding is coarse and the surfaces are frequently delivered in oxidized state. However, the roughness is of short wavelength and when contact occurs the overall dimensions of the contact patch are close to those predicted by a Hertzian elastic case. The local pressures within the contact are higher than that those predicted by Hertz, which assumes a smooth surface elastic contact. The ultrasonic results predict contact pressures some 2.5 times higher. However, it should be noted that the ultrasonic measurement will be an average over the size of the focused spot, so high local peaks at a single asperity contact will not be recorded. The ultrasonic results are therefore useful as a relative comparison but cannot determine asperity scale contact pressures.

As the wheel wears the machining marks are removed and the surface becomes smoother at a local scale. This results in closer conformity and therefore lower contact pressures than the unused case. However, the contact patch is not stationary, in the lateral direction, with respect to either the wheel or the rail surface. This results in an axial distribution of wear and that has the effect of increasing the radius in transverse direction. A larger contact patch and lower contact pressure results.

Contact Pressure Distributions. The ultrasonic measurements can be compared to the numerical modeling of the contact. Figure 17 shows a comparison for the unused specimens at $80 \mathrm{kN}$, also included is the predicted Hertz solution. As shown, there is good global geometric correlation between the ultrasonic results and the numerical model. Where the Hertzian solution gives a continuous pressure distribution, the numerical model agrees with the experimentally determined fragmented contact. This is because the
Table 5 Comparison of ultrasonic measurements and numerical results for the unused specimens

\begin{tabular}{lccc}
\hline \hline Analysis technique & $\begin{array}{c}A_{\text {nom }} \\
\left(\mathrm{mm}^{2}\right)\end{array}$ & $p_{\text {max }}(\mathrm{MPa})$ & $p_{\text {mean }}(\mathrm{MPa})$ \\
\hline Ultrasound & 111.9 & 2628 & 715 \\
Hertzian & 82.9 & 992 & 965 \\
Elastic model & 97.4 & 2278 & 821 \\
Elastic-plastic model & 98.1 & 1500 & 816 \\
\hline \hline
\end{tabular}

boundary element model includes the effects of surface damage and roughness, rather than assuming a perfectly smooth conformal contact. The degree and fragmentation of the contact are qualitatively similar. But on a local level the ultrasonic result and numerical solution differ. This is likely to be due to the difficulty experienced in aligning the surfaces to the same orientation in both the experiment and model.

Table 5 compares the contact pressures from the ultrasonic study to those from the numerical modeling for the unused wheel/ rail contact. As shown, both the elastic and elastic-plastic model solutions predict a higher peak contact pressure than the Hertzian theory. This affect is attributable to the surface roughness fragmenting the contact and unbalancing the overall load distribution. Both the numerical modeling and ultrasonic results have a lower mean contact pressure than theory. This is because the total area of contact in these cases is larger, due to the surface roughening.

For the unused wheel/rail case the numerical elastic model gives the closest agreement. Clearly the surface roughness is such that a smooth surface Hertz analysis under predicts the contact pressure. However, there appears not to be a great deal of plasticity in this case. So replacing the smooth surface assumption with a digitized surface profile gives reasonable agreement. It should be noted that the output of the elastic-plastic model depends strongly on the value of the yield pressure used to truncate the cell pressure matrix.

Figure 18 shows a comparison for the sand damaged wheel/rail specimens at $80 \mathrm{kN}$. Included in Fig. 18 are the ultrasonic pressure map, the Hertzian analytical solution, and the numerical modeling. The elastic numerical result is shown separately at the bottom of the figure; this is because it requires displaying on a different scale.

As shown there is some global correlation between the ultrasonic measurement and the numerical models. It was not possible to exactly match the ultrasonically measured region with the numerically sampled data and calculation. So, the pressure peaks observed by the two methods do not correlate. However, both these results show an extremely fragmented contact with a similar overall size. The overall size of the contact is bigger than that predicted by Hertz due to the sand damage spreading the contact zone. Again the fragmentation of the contact patch is not present in the analytical solution due to the assumption of perfectly smooth conformal contacting surfaces. However, locally the model and experimental result differ. The numerical modeling predicts a defined contact with sharp pressure spikes, whereas the experiment shows a much more graduated pressure profile. Due to this, the model solution has a real area of contact below Hertz, and the experimental result one above. These differences may be due to difficulties in lining the surfaces up at the same relative positions in the experiment and numerical modeling. However, there are also difficulties in modeling the deformation of an extremely fragmented interface.

When comparing contact pressures further problems exist. In the elastic model the contact pressures exceed the hardness of the material, as this is not possible this solution is disregarded. When comparing the ultrasonic result and the elastic-plastic solution, the peak pressures are similar and above the Hertzian predicted value. However, clearly the peak pressure in the elastic-plastic solution is dictated by the yield value used in the analysis, and the threshold 

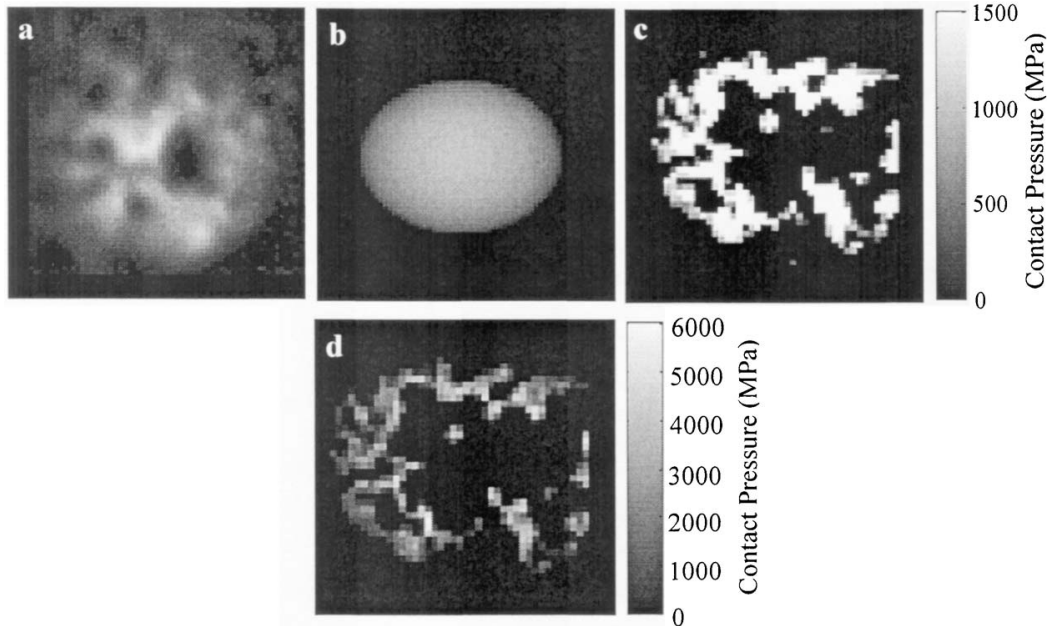

Fig. 18 Damaged specimen contact pressure maps for a load of $\mathbf{8 0 ~} \mathbf{~ k N}$ for (a) ultrasonic measurement, (b) Hertzian smooth elastic, (c) elastic-plastic model, and $(d)$ elastic model

for this is open to argument. Overall, in the sand damaged case correlation is poor, with the only clear result shown by model and experiment being that sand damage fragments the contact.

Toward Dynamic Measurements. The ultrasonic technique presented is best applied to large contacts with gradual pressure variations, like the wheel/rail contact investigated here. The technique is limited by the size of the focused ultrasonic spot. However, by using higher frequency transducers, ultrasonic spots as small as $0.1 \mathrm{~mm}$ in diameter are achievable. Although at these higher frequencies attenuation is increased and less material can be penetrated.

The ultimate aim of this work is to develop the technique to a stage where dynamic measurements can be recorded. Other techniques have been used to study a dynamic wheel/rail contact. Low-pressure air passing through $1 \mathrm{~mm}$ diameter holes drilled into the rail head has been used to measure the contact area as the holes were blocked by the passing wheel [22]. Measurement of these pressure variations allows studying of the contact area shape under dynamic conditions. This, however, can only give very limited spatial data.

With the present ultrasonic technique, obvious difficulties exist when considering its application to dynamic conditions. It would be very difficult to scan a dynamic contact with a single transducer mounted within a water bath. However, advances in array technology offer a solution to these problems. An array of transducers can be created which pulse simultaneously, allowing real time measurement of a complete contact patch. Such an array can then be bonded to the rail in an appropriate position to investigate the dynamic contact.

\section{Conclusions}

- A method has been established to determine interface pressures in a wheel/rail contact. The method uses the measurement of a reflected ultrasonic signal and a parallel calibration procedure.

- Unused, sand damaged, and worn wheel/rail contacts were investigated. The surface roughening in the sand damaged and worn cases was found to significantly influence the pressure distribution. A twin contact was also observed between the worn wheel and rail.

- As the wheel wears it becomes smoother on a short wavelength scale. The contact radius in the transverse direction also increases as wear is distributed axially.
This results in a larger, lower aspect ratio contact patch. Both these effects cause a reduction in measured contact pressure.

- The experimental results for the unused wheel/rail specimens show good agreement with a numerical model of the contact. The model uses boundary element techniques along with real surface data. Results for the sand damaged interface showed a lower level of agreement, this was due to issues when modeling this type of interface.

- The ultrasonic approach is a good method for providing comparative contact stress data. The technique is limited by the resolution of the ultrasonic transducer. Currently contact pressures are averaged over a $1 \mathrm{~mm}$ diameter, which is the focused spot size of the transducer.

\section{References}

[1] Lewis, R., and Dwyer-Joyce, R. S., 2006, "Wear at the Wheel/Rail Interface When Sanding is Used to Increase Adhesion,” Proc. Inst. Mech. Eng., F J. Rail Rapid Transit, 220, pp. 29-41.

[2] Telliskivi, T., and Olofsson, U., 2001, "Contact Mechanics Analysis of Measured Wheel-Rail Profiles using the Finite Element Method," Proc. Inst. Mech Eng., F J. Rail Rapid Transit, 215, pp. 65-72.

[3] Yan, W., and Fischer, F. D., 2000, "Applicability of the Hertz Contact Theory to Rail-Wheel Contact Problems," Arch. Appl. Mech., 70, pp. 255-268.

[4] Dwyer-Joyce, R. S., and Drinkwater, B. W., 1998, "Analysis Of Contact Pressure Using Ultrasonic Reflection," Experimental Mechanics, Proceedings of the 11th Annual Conference on Experimental Mechanics, Balkema, Rotterdam, pp. 747-754.

[5] Pau, M., Aymerich, F., and Ginesu, F., 2000, "Ultrasonic Measurement of Nominal Contact Area and Contact Pressure in a Wheel/Rail System," Proc. Inst. Mech. Eng., F J. Rail Rapid Transit, 214, pp. 231-243.

[6] Kendall, K., and Tabor, D., 1971, "An Ultrasonic Study of the Area of Contact between Stationary and Sliding Surfaces," Proc. R. Soc. London, Ser. A, 323, pp. 321-340.

[7] Tattersall, A. G., 1973, "The Ultrasonic Pulse-Echo Technique as Applied to Adhesion Testing," J. Phys. D, 6, pp. 819-832.

[8] Drinkwater, B. W., Dwyer-Joyce, R. S., and Cawley, P., 1996, "A Study of the Interaction between Ultrasound and a Partially Contacting Solid-Solid Interface," Proc. R. Soc. London, Ser. A, 452, pp. 2613-2628.

[9] Hodgson, K., Dwyer-Joyce, R. S., and Drinkwater, B. W., 2000, "Ultrasound as an Experimental Tool for Investigating Engineering Contacts," Proceedings of the 9th Nordic Symposium on Tribology, P. Andersson, H. Ronkainen, and K. Holmberg, eds., Vol. 2, pp. 377-386.

[10] Arakawa, T., 1983, "A Study of the Transmission of Elastic Waves by a Periodic Array of Cracks," Mater. Eval., 41, pp. 714-719.

[11] Krautkrämer, J., and Krautkrämer, H., 1990, Ultrasonic Testing of Materials, Springer Verlag, Berlin. 
[12] Olofsson, U., and Telliskivi, T., 2003, "Wear, Friction and Plastic Deformation of Two Rail Steels-Full Scale Test and Laboratory Study," Wear, 254, pp. 80-93.

[13] Olofsson, U., and Nilsson, R., 2002, "Surface Cracks and Wear of Rail: A Full Scale Test and Laboratory Study,” Proc. Inst. Mech. Eng., F J. Rail Rapid Transit, 216, pp. 249-264.

[14] Fischer, G., Grubisic, V., and Widmayer, H., 1988, "Fatigue Tests on Wheelsets under Simulated Service Strength Spectra," Proceedings of the 9th International Wheelset Congress, Montreal.

[15] Dwyer-Joyce, R. S., Drinkwater, B. W., and Quinn, A. M., 2001, "The Use of Ultrasound in the Investigation of Rough Surface Interfaces," ASME J. Tribol., 123, pp. 8-16.

[16] Krolikowski, J., and Szczepek, J., 1991, "Prediction of Contact Parameters using Ultrasonic Method," Wear, 148, pp. 181-195.

[17] Johnson, K. L., 1985, Contact Mechanics, Cambridge University Press,
Cambridge.

[18] Tabor, D., 1951, The Hardness of Metals, Monographs on the Physics and Chemistry of Materials, Oxford University Press, Oxford.

[19] Björklund, S., 1995, "Elastic Contacts between Rough Surfaces," Doctoral Thesis, Department of Machine Design, KTH, Report No. TRITA-MMK 1995:4.

[20] Love, A. E. H., 1929, "Stress Produced in an Infinite Solid by Pressure on Part of the Boundary," Philos. Trans. R. Soc. London, Ser. A, A228(377), pp. 54-59.

[21] Webster, M. N., and Sayles, R. S., 1986, "A Numerical Model for the Elastic Frictionless Contact of Real Rough Surfaces," Trans. ASME, J. Tribol., 108(3), pp. 314-320.

[22] Poole, W., 1987, "The Measurement of Contact Area between Opaque Objects Under Static and Dynamic Rolling Conditions," Proceedings of Contact Mechanics and Wear of the Wheel/Rail System, University of Rhode Island, Waterlooville Press, pp. 59-72. 